Research Article

\title{
The average domination polynomial of graphs is unimodal
}

\author{
Mohammad Reza Oboudi* \\ Department of Mathematics, College of Sciences, Shiraz University, Shiraz, 71457-44776, Iran
}

(Received: 23 July 2021. Received in revised form: 25 August 2021. Accepted: 25 August 2021. Published online: 27 August 2021.$)$

(c) 2021 the author. This is an open access article under the CC BY (International 4.0) license (www.creativecommons.org/licenses/by/4.0/).

\begin{abstract}
Let $G$ be a simple graph on $n$ vertices. A dominating set of $G$ is a subset of the vertex set $V(G)$ of $G$, say $S$, such that every vertex in $V(G) \backslash S$ is adjacent to at least one vertex of $S$. The domination polynomial of $G$ is the polynomial $D(G, x)=$ $\sum_{i=1}^{n} d(G, i) x^{i}$, where $d(G, i)$ is the number of dominating sets of $G$ of size $i$. For every $n \geq 1$, let $\Phi_{n}(x)$ be the average of the domination polynomials of all labeled graphs on $n$ vertices. In this paper, the polynomial $\Phi_{n}(x)$ is studied and it is shown that $\Phi_{n}(x)$ is log-concave and unimodal.
\end{abstract}

Keywords: dominating set; domination polynomial; unimodal polynomial; log-concave polynomial.

2020 Mathematics Subject Classification: 05C31, 05C69, 05C70.

\section{Introduction}

Throughout this paper, we consider only simple graphs (the graphs with no loops and multiple edges). Let $G=(V(G), E(G))$ be a simple graph. The order of $G$ is the number of vertices of $G$. For a vertex $v \in V(G)$, the degree of $v$ is the number of edges incident with $v$ and is denoted by $\operatorname{deg}_{G}(v)$ (for simplicity we write $\operatorname{deg}(v)$ instead of $\operatorname{deg}_{G}(v)$ ). For a vertex $v \in V(G)$, the open neighborhood of $v$ is the set $N(v)=\{u \in V: u v \in E(G)\}$ and the closed neighborhood is the set $N[v]=N(v) \cup\{v\}$. For a set $S \subseteq V(G)$, the open neighborhood of $S$ is $N(S)=\bigcup_{v \in S} N(v)$ and the closed neighborhood of $S$ is $N[S]=N(S) \cup S$. A set $S \subseteq V(G)$ is a dominating set of $G$ if $N[S]=V(G)$, or equivalently, every vertex in $V(G) \backslash S$ is adjacent to at least one vertex of $S$. The domination number of $G$, denoted by $\gamma(G)$, is the minimum of the cardinality of the dominating sets of $G$. We denote the complete graph of order $n$, the cycle of order $n$ and the path of order $n$, by $K_{n}, C_{n}$, and $P_{n}$, respectively.

There are numerous polynomials associated with graphs. For example chromatic polynomial, clique polynomial, independence polynomial, matching polynomial, edge cover polynomial, edge elimination polynomial, domination polynomial and Tutte polynomial. For more details, see [1]- [22] and references therein. By studying these polynomials one can obtain some properties of a graph. For instance the roots of these polynomials reflect some important information about the structure of graphs. Let $G$ be a graph of order $n$. The domination polynomial of $G$ that is denoted by $D(G, x)$ is the one variable polynomial such that the coefficient of $x^{k}$ is $d_{k}$, where $d_{k}$ is the number of dominating sets of $G$ with size $k$. More precisely

$$
D(G, x)=\sum_{k=1}^{n} d_{k} x^{k}
$$

For example, the domination polynomial of the complete graph $K_{n}$ is $D\left(K_{n}, x\right)=(x+1)^{n}-1$. The domination polynomial was first introduced in [5, 8].

One of the most interesting properties of graph polynomials is unimodality. A polynomial $f(x)=\sum_{i=0}^{n} a_{i} x^{i}$ with real coefficients (or a sequence $\left(a_{0}, \ldots, a_{n}\right)$ ) is called unimodal if there is $k \in\{0, \ldots, n\}, k$ is called the mode of $f(x)$, such that

$$
a_{0} \leq \cdots \leq a_{k-1} \leq a_{k} \geq a_{k+1} \geq \cdots \geq a_{n}
$$

Also, $f(x)$ (or a sequence $\left(a_{0}, \ldots, a_{n}\right)$ ) is called logarithmically concave (or simply, log-concave), if for every $1 \leq i \leq n-1$, $a_{i}^{2} \geq a_{i-1} a_{i+1}$. The polynomial $f(x)$ (or a sequence $\left(a_{0}, \ldots, a_{n}\right)$ ) is called symmetric (or palindromic) if $a_{i}=a_{n-i}$ for $i=0,1, \ldots, n$. It is known that any log-concave polynomial with positive coefficients (or a sequence of positive numbers) is also unimodal. See [21] for more details on these definitions.

*E-mail addresses: mr_oboudi@yahoo.com,mr_oboudi@shirazu.ac.ir 
The unimodality problems of graph polynomials have always been of great interest to researchers in graph polynomials. In [20], it has been conjectured that the chromatic polynomial of a graph is unimodal. Recently, in [14], this conjectured has been proved. It is conjectured that the domination polynomial of every graph is unimodal [5]. Also, there is a famous conjecture due to Alavi et al. [1] on the unimodality of the independence polynomial of trees. The unimodality of graph polynomials, in particular the unimodality of independence polynomial, have been extensively studied, see [1, 7,10,11,14, 16,22]. In [11], the authors show that the average of the independence polynomial of graphs is unimodal. Motivated by these papers, in this paper we study the unimodality of domination polynomial. For every $n \geq 1$, let $\Phi_{n}(x)$ be the average of the domination polynomials of all labeled graphs on $n$ vertices. In this paper first we indicate $\Phi_{n}(x)$ and then show that this polynomial is log-concave. Since the coefficients of $\Phi_{n}(x)$ are positive, we conclude that $\Phi_{n}(x)$ is unimodal.

\section{Results}

For every integer $n \geq 1$, let $\mathcal{G}_{n}$ be the set of all simple graphs on the vertices $v_{1}, \ldots, v_{n}$. In other words $\mathcal{G}_{n}$ is the set of all

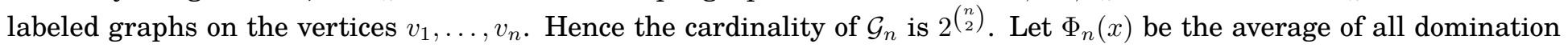
polynomial of graphs of $\mathcal{G}_{n}$. In other words,

$$
\Phi_{n}(x)=2^{-\left(\begin{array}{l}
n \\
2
\end{array}\right)} \sum_{G \in \mathcal{G}_{n}} D(G, x) .
$$

For example, $\Phi_{1}(x)=x, \Phi_{2}(x)=x+x^{2}, \Phi_{3}(x)=\frac{3 x+9 x^{2}+4 x^{3}}{4}$ and $\Phi_{4}(x)=\frac{4 x+27 x^{2}+28 x^{3}+8 x^{4}}{8}$. In this section, we determine the coefficients of polynomial $\Phi_{n}(x)$ and show that $\Phi_{n}(x)$ is unimodal (in fact we prove that this polynomial is log-concave).

Theorem 2.1. For every integer $n \geq 1$ we have

$$
\Phi_{n}(x)=\sum_{k=1}^{n}\left(\begin{array}{l}
n \\
k
\end{array}\right)\left(1-2^{-k}\right)^{n-k} x^{k}
$$

Proof. Let $n \geq 1$ be an integer and

$$
S_{n}(x)=2^{\left(\begin{array}{c}
n \\
2
\end{array}\right)} \Phi_{n}(x) .
$$

For a graph $G$, let $T(G)$ be the set of all dominating sets of $G$. Therefore $D(G, x)=\sum_{I \in T(G)} x^{|I|}$. Note that the summation is taken over all dominating sets of $G$. Hence

$$
S_{n}(x)=\sum_{G \in \mathcal{G}_{n}} D(G, x)=\sum_{G \in \mathcal{G}_{n}} \sum_{I \in T(G)} x^{|I|} .
$$

Therefore

$$
S_{n}(x)=\sum_{\varnothing \neq I \subseteq\left\{v_{1}, \ldots, v_{n}\right\}} \lambda_{I} x^{|I|},
$$

where $\lambda_{I}$ is the number of graphs $G$ with the vertex set $\left\{v_{1}, \ldots, v_{n}\right\}$ such that $I$ is a dominating set of $G$.

Hence to complete the proof it suffices to find $\lambda_{I}$. For every $1 \leq k \leq n$, let $I_{k}=\left\{v_{1}, \ldots, v_{k}\right\}$. Since the number of graphs

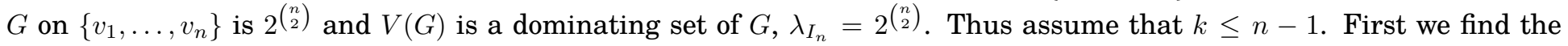
number of bipartite graphs $H$ with the parts $\left\{v_{1}, \ldots, v_{k}\right\}$ and $\left\{v_{k+1}, \ldots, v_{n}\right\}$ such that the degree of all vertices $v_{k+1}, \ldots, v_{n}$ in $H$ is non-zero. In other words $N\left(v_{i}\right) \cap\left\{v_{1}, \ldots, v_{k}\right\} \neq \emptyset$ for $i=k+1, \ldots, n$. For $j=1, \ldots, n-k$ define the property $c_{j}$ as "the degree of $v_{j+k}$ is zero". Let $M\left(c_{j_{1}} c_{j_{2}} \cdots c_{j_{m}}\right)$ be the number of bipartite graphs $T$ with the parts $\left\{v_{1}, \ldots, v_{k}\right\}$ and $\left\{v_{k+1}, \ldots, v_{n}\right\}$ such that the degree of all vertices $v_{k+j_{1}}, \ldots, v_{k+j_{m}}$ in $T$ is zero. Therefore $M\left(c_{j_{1}} c_{j_{2}} \cdots c_{j_{m}}\right)=2^{k(n-k-m)}$ (note that the number of bipartite graphs $L$ with parts $X$ and $Y$ is $2^{|X||Y|}$ ). Let $\beta$ be the number of bipartite graphs $H$ with the parts $\left\{v_{1}, \ldots, v_{k}\right\}$ and $\left\{v_{k+1}, \ldots, v_{n}\right\}$ such that the degree of all vertices $v_{k+1}, \ldots, v_{n}$ in $H$ is non-zero. Using the inclusion - exclusion principle we conclude that

$$
\beta=2^{k(n-k)}-\sum_{1 \leq j \leq n-k} M\left(c_{j}\right)+\sum_{1 \leq j<j^{\prime} \leq n-k} M\left(c_{j} c_{j^{\prime}}\right)-\sum_{1 \leq j<j^{\prime}<j^{\prime \prime} \leq n-k} M\left(c_{j} c_{j^{\prime}} c_{j^{\prime \prime}}\right)+\cdots+(-1)^{n-k} M\left(c_{1} \cdots c_{n-k}\right) .
$$

Therefore

$$
\beta=2^{k(n-k)}-\left(\begin{array}{c}
n-k \\
1
\end{array}\right) 2^{k(n-k-1)}+\left(\begin{array}{c}
n-k \\
2
\end{array}\right) 2^{k(n-k-2)}+\cdots+(-1)^{n-k}\left(\begin{array}{c}
n-k \\
n-k
\end{array}\right) 2^{k(n-k-(n-k))} .
$$

Hence by the binomial Theorem we obtain that

$$
\beta=\sum_{j=0}^{n-k}(-1)^{j}\left(\begin{array}{c}
n-k \\
j
\end{array}\right) 2^{k(n-k-j)}=\sum_{j=0}^{n-k}(-1)^{j}\left(\begin{array}{c}
n-k \\
j
\end{array}\right)\left(2^{k}\right)^{n-k-j}=\left(2^{k}-1\right)^{n-k} .
$$


Since the number of graphs on $\left\{v_{1}, \ldots, v_{k}\right\}$ is $2^{\left(\begin{array}{c}k \\ 2\end{array}\right)}$ and the number of graphs on $\left\{v_{k+1}, \ldots, v_{n}\right\}$ is $\left.2^{\left({ }^{n-k}\right.}\right)^{(2)}$ we find that

$$
\lambda_{I_{k}}=2^{\left(\begin{array}{c}
k \\
2
\end{array}\right) 2} 2^{\left(\begin{array}{c}
n-k \\
2
\end{array}\right)} \beta \text {. }
$$

On the other hand

$$
\left(\begin{array}{l}
n \\
2
\end{array}\right)=\left(\begin{array}{c}
k \\
2
\end{array}\right)+\left(\begin{array}{c}
n-k \\
2
\end{array}\right)+\left(\begin{array}{c}
k \\
1
\end{array}\right)\left(\begin{array}{c}
n-k \\
1
\end{array}\right)
$$

So by the Equations (2), (3) and (4) we obtain that

$$
\lambda_{I_{k}}=2^{\left(\begin{array}{c}
n \\
2
\end{array}\right)-k(n-k)}\left(2^{k}-1\right)^{n-k}=2^{\left(\begin{array}{c}
n \\
2
\end{array}\right)} \frac{\left(2^{k}-1\right)^{n-k}}{\left(2^{k}\right)^{n-k}}=2^{\left(\begin{array}{c}
n \\
2
\end{array}\right)}\left(1-2^{-k}\right)^{n-k} .
$$

This equality shows that for every $I$ where $\emptyset \neq I \subseteq\left\{v_{1}, \ldots, v_{n}\right\}$,

$$
\lambda_{I}=2^{\left(\begin{array}{c}
n \\
2
\end{array}\right)}\left(1-2^{-|I|}\right)^{n-|I|} .
$$

On the other hand the number of subset of $\left\{v_{1}, \ldots, v_{n}\right\}$ with cardinality $k$ is $\left(\begin{array}{l}n \\ k\end{array}\right)$. So, by Equations (1) and (6) we conclude that the coefficient of $x^{k}$ in $S_{n}(x)$ is

$$
\left(\begin{array}{l}
n \\
k
\end{array}\right) 2^{\left(\begin{array}{l}
n \\
2
\end{array}\right)}\left(1-2^{-k}\right)^{n-k}
$$

for $k=0, \ldots, n$. In other words, the coefficient of $x^{k}$ in $\Phi_{n}(x)$ is

$$
\left(\begin{array}{l}
n \\
k
\end{array}\right)\left(1-2^{-k}\right)^{n-k}
$$

The proof is complete.

Now we show that the average of the domination polynomials of graphs is unimodal.

Theorem 2.2. For every integer $n \geq 1$ the polynomial $\Phi_{n}(x)$ is log-concave and so is unimodal.

Proof. Let $n \geq 1$ be an integer. The result easily follows for $n \leq 2$. Hence let $n \geq 3$. For every $k \in\{0,1, \ldots, n\}$ let $A_{n, k}=\left(1-2^{-k}\right)^{n-k}$. Note that $A_{n, 0}=0$ and $A_{n, k}>0$ for $k \geq 1$. We claim the sequence $A_{n, 0}, \ldots, A_{n, n}$ is log-concave. In other words for every $k \in\{1, \ldots, n-1\}, A_{n, k}^{2} \geq A_{n, k-1} A_{n, k+1}$. For $k=1$ there is nothing to prove. So let $k \geq 2$. Since $\left(2^{k}-1\right)^{2}>\left(2^{k-1}-1\right)\left(2^{k+1}-1\right)$, we have $\left(2^{k}-1\right)^{2 n-2 k}>\left(2^{k-1}-1\right)^{n-k}\left(2^{k+1}-1\right)^{n-k}$. On the other hand $2^{k+1}-1>4\left(2^{k-1}-1\right)$. Hence

$$
\left(2^{k}-1\right)^{2 n-2 k}>\frac{2^{k+1}-1}{2^{k-1}-1}\left(2^{k-1}-1\right)^{n-k+1}\left(2^{k+1}-1\right)^{n-k-1}>4\left(2^{k-1}-1\right)^{n-k+1}\left(2^{k+1}-1\right)^{n-k-1} .
$$

This implies that

$$
\left(2^{k}-1\right)^{2 n-2 k}>4\left(2^{k-1}-1\right)^{n-k+1}\left(2^{k+1}-1\right)^{n-k-1} .
$$

On the other hand

$$
2 k(n-k)=(k-1)(n-k+1)+(k+1)(n-k-1)+2 .
$$

By dividing the sides of Equation (7) by $2^{2 k(n-k)}$ and using Equation (8), we obtain

$$
\left(\left(\frac{2^{k}-1}{2^{k}}\right)^{n-k}\right)^{2}>\left(\frac{2^{k-1}-1}{2^{k-1}}\right)^{n-k+1}\left(\frac{2^{k+1}-1}{2^{k+1}}\right)^{n-k-1}
$$

This shows that

$$
\left(\left(1-2^{-k}\right)^{n-k}\right)^{2}>\left(1-2^{-(k-1)}\right)^{n-k+1}\left(1-2^{-(k+1)}\right)^{n-k-1} .
$$

Hence the claim is proved. Note that the positive sequence

$$
\left(\begin{array}{l}
n \\
0
\end{array}\right),\left(\begin{array}{l}
n \\
1
\end{array}\right), \ldots,\left(\begin{array}{l}
n \\
n
\end{array}\right)
$$

is log-concave. Thus (since the non-negative sequence $A_{n, 0}, \ldots, A_{n, n}$ is log-concave) we conclude that the sequence

$$
\left(\begin{array}{c}
n \\
0
\end{array}\right) A_{n, 0},\left(\begin{array}{c}
n \\
1
\end{array}\right) A_{n, 1}, \ldots,\left(\begin{array}{c}
n \\
n
\end{array}\right) A_{n, n}
$$

is also log-concave. In other words $\Phi_{n}(x)$ is log-concave. Since the coefficients of $\Phi_{n}(x)$ are positive, $\Phi_{n}(x)$ is unimodal. The proof is complete. 


\section{References}

[1] Y. Alavi, P. J. Malde, A. J. Schwenk, P. Erdős, The vertex independence sequence of a graph is not constrained, Congr. Numer. 58 (1987) 15-23.

[2] S. Akbari, S. Alikhani, Y. H. Peng, Characterization of graphs using domination polynomial, European J. Combin. 31 (2010) $1714-1724$.

[3] S. Akbari, M. R. Oboudi, Cycles are determined by their domination polynomials, Ars Combin. 116 (2014) 353-358.

[4] S. Akbari, M. R. Oboudi, On the edge cover polynomial of a graph, European J. Combin. 34 (2013) 297-321.

[5] S. Alikhani, Dominating Sets and Domination Polynomials of Graphs, Ph.D. Dissertation, University Putra Malaysia, Seri Kembangan, 2008.

[6] S. Alikhani, J. I. Brown, S. Jahari, On the domination polynomials of friendship graphs, Filomat 30 (2016) 169-178.

[7] S. Alikhani, F. Jafari, On the unimodality of independence polynomial of certain classes of graphs, Trans. Comb. 2 (2013) 33-41.

[8] S. Alikhani, Y. H. Peng, Introduction to Domination Polynomial of a Graph, Ars Combin. 114 (2014) 257-266.

[9] I. Averbouch, B. Godlin, J. A. Makowsky, An extension of the bivariate chromatic polynomial, European J. Combin. 31 (2010) 1-17.

[10] P. Bahls, N. Salazar, Symmetry and unimodality of independence polynomials of path-like graphs, Australas. J. Combin. 47 (2010) $165-175$.

[11] J. I. Brown, R. J. Nowakowski, Average independence polynomials, J. Combin. Theory Series B 93 (2005) 313-318.

[12] P. Csikvari, M. R. Oboudi, On the roots of edge cover polynomials of graphs, European J. Combin. 32 (2011) 1407-1416.

[13] K. Dohmen, P. Tittmann, Domination reliability, Electron. J. Combin. 19 (2012) Art\# P15.

[14] J. Huh, Milnor numbers of projective hypersurfaces and the chromatic polynomial of graphs, J. Amer. Math. Soc. 25 (2012) 907-927.

[15] T. Kotek, J. Preen, P. Tittmann, Subset-sum representations of domination polynomials, Graphs Combin. 30 (2014) 647-660.

[16] M. R. Oboudi, On the number of perfect matchings of generalized theta graphs and the edge cover polynomials of friendship graphs, Filomat 33 (2019) 111-120.

[17] M. R. Oboudi, Some results on the independence polynomial of unicyclic graphs, Discuss. Math. Graph Theory 38 (2018) 515-524.

[18] M. R. Oboudi, On the largest real root of independence polynomials of trees, Ars Combin. 137 (2018) 149-164.

[19] M. R. Oboudi, On the roots of domination polynomial of graphs, Discrete Appl. Math. 205 (2016) 126-131.

[20] R. C. Read, An introduction to chromatic polynomials, J. Combin. Theory 4 (1968) 52-71.

[21] R. P. Stanley, Log-concave and unimodal sequences in algebra, combinatorics and geometry, Ann. New York Acad. Sci. 576 (1989) 500-534.

[22] Y. Wang, B. X. Zhu, On the unimodality of independence polynomials of some graphs, European J. Combin. 32 (2011) 10-20. 